
шаг впереА, Ава шага назаА

\author{
Ф.Ф. Аптикаев (1D \\ ФБГУН Институт физики Земли РАН, Россия, 123242, \\ Москва, ул. Большая Грузинская, 10, стр. 1, e-mail: felix@ ifz.ru \\ Статья поступила: 21.04.2020, доработана: 21.05.2020, одобрена в печать: 24.05.2020
}

Резюме: Актуальность работы. На основании эмпирических данных показано, что отечественные строительные нормы и правила существенно занижают уровень расчетных сейсмических воздействий. В этом кроется аномально большое количество жертв и огромный ущерб при землетрясениях в нашей стране по сравнению с США и Японией. При этом в проекте СП 14.133302019 года наблюдается новое снижение уровня воздействий. Сравнение нормативных уровней ускорения различных стран показало, что наши расчетные ускорения самые низкие. Например, уровень расчетных ускорений в наших нормах по сравнению с нормами США в три раза ниже. Цель работы - показать реальное соотношение сейсмической интенсивности в баллах и ускорения грунта и найти параметры сейсмического движения грунта, наиболее тесно коррелируемые со степенью повреждения зданий и сооружений. Методы исследования - статистический анализ записей сильных движений, сравнение методов задания сейсмических воздействий в строительных нормах различных стран. Результаты работы - показано, что задание уровня ускорений в отечественных нормах занижено примерно вдвое и не соответствует допустимым уровням превышения воздействий в баллах на картах ОСР. Ускорения не являются достаточной характеристикой сейсмических воздействий. Наилучшими характеристикоами сейсмических воздействий являются энергетические. Важным фактором занижения воздействий является использование вместо реальных значений ускорений так называемых «эфрфективных» ускорений, которые в результате фрильтрации ниже реальных примерно в полтора раза. Причем приведенный выше термин не применяется. И многие проектировщики считают, что в нормах приведены реальные пиковые ускорения. В шкале MSK-64, которая до сих пор используется в наших нормах, надежные оценки связи сейсмической интенсивности с ускорениями были получены только для 6 баллов. Для более высоких интенсивностей значения ускорений были получены экстраполяцией при предположении об удвоении амплитуды на балл. В настоящее время обильный эмпирический материал показал несостоятельность этого предположения. Это привело к дополнительному занижению расчетной амплитуды ускорений, особенно для 9 баллов. При применении спектрального метода этот недостаток частично компенсируется другими предположениями. Но для динамического метода расчетов применение эффективных значений приводит к большим ошибкам. Не принимаются во внимание достижения других стран. Например, за рубежом при расчете зданий и сооружений на сейсмостойкость учитывается продолжительность колебаний (модель Ариаса), а в наших нормах этот важный параметр полностью игнорируется, хотя в сейсмической шкале ГОСТ Р ГОСТ Р 57546-2017 оценки, соответствующие модели Ариаса, имеются. Отмечаются разногласия в строительных нормах различных организаций и ведомств нашей страны. В тексте нормативнвых документов встречаются серьезные противоречия. Например, частотный состав обобщенного спектра задается в одной полосе частот, а уровень спектра - в другой. Карты ОСР предполагают использование ускорений, близких к шкале ГОСТ Р ГОСТ Р 57546-2017, а нормы требуют использование шкалы MSK-64. Шкала MSK-64 уже давно не используется на практике, поскольку в ней нет описания панельных зданий, крупноблочных зданий, зданий с сейсмоусилением и вообще зданий выше 6 этажей. Вместо среднестатистического значения коэффициента динамического усиления используется значение, полученное с нарушением всех правил обработки эмпирического материала. Наконец, карты ОСР строятся для условия превышения воздействий до 1\% (карта ОСР-С), а ускорения для этого случая - с вероятностью превышения $99 \%$. 
Ключевые слова: Строительные нормы, сейсмические воздействия, сейсмическая интенсивность, ускорение, скорость колебаний, мощность колебаний, продолжительность колебаний, коэффициент динамического усиления.

Для цитирования: Аптикаев Ф.Ф. Новые строительные нормы: шаг вперед, два шага назад. Геология и геофизика Юга России. 2020. 10(2): 71-81. DOI: 10.46698/VNC.2020.50.57.005.

\title{
Original paper \\ New Building Codes: one step forward, two steps backward
}

\author{
F.F. Aptikaev \\ Schmidt Institute of Physics of the Earth of the Russian Academy of Sciences, 10/1 Bolshaya \\ Gruzinskaya, Moscow 123242, Russian Federation, e-mail: felix@ifz.ru
}

Received: 21.04.2020, revised: 21.05.2020, accepted: 24.05.2020

\begin{abstract}
Relevance. Based on empirical data, it is shown that domestic building codes and rules significantly underestimate the level of design seismic effects. This is the reason for an abnormally large number of victims and huge damage during earthquakes in our country compared to the United States and Japan. At the same time, in the project SP14.13330 2019, a new decrease in the level of seismic effects is observed. A comparison of the normative acceleration levels of different countries showed that our calculated accelerations are the lowest. For example, the level of calculated acceleration in our standards is three times lower than in the United States. The aim of the work is to show the real correlation of seismic intensity in points and soil acceleration and find the parameters of seismic ground motion that are most closely correlated with the degree of damage to buildings and structures. Methods - statistical analysis of strong motion records, comparison of methods for setting seismic effects in building codes of different countries. Results. It is shown that setting the level of acceleration in domestic standards is underestimated by about half and does not correspond to the permissible levels of exceeding the effects in points on the GSZ maps. Acceleration is not a sufficient characteristic of seismic effects. The best characteristic of seismic effects is energy. An important factor in underestimating the effects is the use of so-called "effective" accelerations instead of real values of accelerations, which are about one and a half times lower than real ones as a result of filtering. Moreover, the above term is not applied. And many designers believe that the standards show real peak accelerations. In the MSK-64 scale, which is still used in our standards, reliable estimates for the relationship of seismic intensity with accelerations were obtained only for 6 points. For higher intensities, the acceleration values were obtained by extrapolation under the assumption that the amplitude was doubled per point. At present, abundant empirical material has shown the failure of this assumption. This led to an additional underestimation of the calculated amplitude of accelerations, especially for 9 points. When applying the spectral method, this drawback is partially compensated by other assumptions. But for the dynamic calculation method, the use of effective values leads to large errors. The achievements of other countries are not taken into account. For example, abroad, when calculating buildings and structures for earthquake resistance, the duration of vibrations (Arias model) is taken into account, and in our standards this important parameter is completely ignored, although in the seismic scale GOST R GOST R 57546-2017, estimates corresponding to the Arias model are available. Disagreements are noted in the building codes of various organizations and departments of our country. Serious contradictions occur in the text of regulatory documents. For example, the frequency composition of the generalized spectrum is specified in one frequency band, and the spectrum level in another. GSZ maps require the use of accelerations close to the GOST R GOST R 57546-2017 scale, and norms require the use of the MSK-64 scale. The MSK-64 scale has not been used in practice for a long time, since it does not contain a description of panel buildings, large-block buildings, buildings with seismic retrofitting, and generally buildings above 6 floors. Instead of the average value of the dynamic amplification coefficient, the value
\end{abstract}


obtained in violation of all the rules for processing empirical material is used. Finally, the GSZ maps are built for the condition that the impacts are exceeded up to 1\% (GSZ-S map), and accelerations for this case - with a probability of exceeding $99 \%$.

Keywords: Building codes, seismic treatment, seismic intensity, acceleration, vibration velocity, power, duration, coefficient of dynamic amplification.

For citation: Aptikaev F.F. New Building Codes: one step forward, two steps backward. Geologiya I Geofizika Yuga Rossii = Geology and Geophysics of Russian South. (in Russ.). 2020. 10(2): 71-81. DOI: 10.46698/VNC.2020.50.57.005.

\section{Введение}

С 1981 года ведутся бесплодные попытки улучшить строительные нормы. То что наши нормы не соответствуют реальной сейсмической опасности не вызывает сомнений. В США за последние 100 лет при 21 разрушительном землетрясении погибло в общей сложности 264 человека [Jaiswal et al., 2011]. Например, при землетрясении Империал Вэлли в 1979 году с магнитудой 6,5 жертв не было, при землетрясении Лома Приета в 1989 году с магнитудой 7,1, происшедшем в густонаселенном районе, погибли 62 человека, при землетрясении Нортридж 1994 года с магнитудой 6,7 погибли 57 человек. Аналогичная картина наблюдается в Японии. При землетрясении Тюэцу (Япония) 2004 года с магнитудой 6,7 погибли 40 человек, при землетрясении Фукусима (Япония) 2011 года с магнитудой 6,6 погибли 7 человек. В строительных нормах США сейсмические воздействия задаются на основании представительного количества записей сильных движений. Практически для каждого активного разлома записи сильных движений имеются. Наши нормы не опираются на эмпирический материал и воздействия задаются на ряде допущений и предположений. Нормам 1981 года [СНИП-II-7-81*, 2013], которые являются основой всех последующих версий, соответствуют следующие эффекты: в Спитаке (Армения) при землетрясении 1988 года с магнитудой 6,8 погибло 25 тысяч человек, при землетрясении в Нефтегорске 1995 года с магнитудой 7,6 погибло 2240 человек - большинство населения маленького городка. В чем причина наших потерь? Напрашивается наиболее вероятная причина - несоответствие принятого в нормах уровня воздействий реальным значениям. Нормы оперируют уровнями ускорений по шкале MSK-64 [Медведев, 1968]. Насколько надежны оценки расчетных ускорений, приведенных в шкале MSK-64? Сами значения, округленные до двух знаков, наводят на предположение о грубости оценок. В таблице 1 приведены количества записей ускорений, использованных при построении шкал MSK-64 и шкалы [ГОСТ Р 57546-2017].

Таблий 1. / Table 1.

Количество записей сильных движений, которые использованы при составлении инструментальных шкал сейсмической интенсивности. / The number of strong movements records used in compiling instrumental scales of seismic intensity.

\begin{tabular}{|l|c|c|c|c|}
\hline \multicolumn{1}{|c|}{ I, баллы / I, points } & $\mathbf{9}$ & $\mathbf{8}$ & $\mathbf{7}$ & $\mathbf{6}$ \\
\hline MSK-64, 1964 & 2 & 5 & 13 & 65 \\
\hline ГOCT P 57546-2017 / GOST R 57546-2017 & 104 & 172 & 212 & 353 \\
\hline
\end{tabular}




\section{Цель работы}

Ясно, что достаточно надежные оценки в 1964 году получены только для интенсивности $I=6$. Для более высоких интенсивностей оценки производились путем экстраполяции при предположении об удвоении амплитуды на балл. Такое предположение было обосновано тем, что реально наблюдавшиеся ускорения в диапазоне 1-1000 cм/ $\mathrm{c}^{2}$ были отнесены к диапазону интенсивностей 2-12 баллов. Однако накопленные эмпирические данные показали, что максимальные ускорения достигаются при 9 баллах и при дальнейшем росте интенсивности уже не изменяются [Campbell, 1981; Bureau, 1981; Hanks, Johnson, 1976; Yamada et al., 1997; Bommer, Martinez-Pereira, 2000]. В работе [Bommer, Martinez-Pereira, 2000] на эмпирическом материале показано, что при 9 и 11 баллах ускорения одинаковы. Не нужно быть большим специалистом в области механики горных пород, чтобы сообразить, что деформация, которую могут выдержать горные породы, должна иметь предел, не зависящий от масштаба явления, т.е. от магнитуды землетрясения. Независимость безразмерных величин от масштаба явления следует из теории размерностей и подобия [Седов, 1987]. Следовательно, интервалу ускорений 1-1000 cм/ $\mathrm{c}^{2}$ соответствует интервал интенсивности 2-9 баллов, т.е. изменению ускорений в 2,5 раза на балл, что подтверждается и прямыми оценками этого соотношения [Аптикаев и др., 2008] (рис. 1). В нормах интенсивностям 7, 8, 9 баллам соответствуют ускорения $100,200,400 \mathrm{~cm} / \mathrm{c}^{2}$ соответственно. Пользователи воспринимают эти значения, как наиболее вероятные и используют их. Между тем, приведенные значения получены путем фильтрации в полосе 2-5 Гц.

\section{Методы исследования}

В США такие значения называют «эффективными». У нас этим термином не пользуются, что и привело к недоразумениям. По оценке автора шкалы С.В. Медведева фильтрация привела к занижению уровня ускорений примерно в полтора раза [Медведев, 1978]. К такому же выводу пришел редактор карты ОСР-97 В.И. Уломов, оценивший значения уровня ускорений в шкале MSK-64 как значения нижнего уровня диапазона интенсивности [Уломов, 1994]. Занижение уровня колебаний в шкале MSK-64 связано и с другими факторами: например, пересчет смещений в ускорения при предположении о синусоидальной форме колебаний. Метод оценки сейсмической опасности, разработанный для карты ОСР-97, использовался в международном проекте GSHAP при построении карт сейсмической опасности мира, куда входит и карта ОСР-97. Поэтому карта ОСР-97 была построена и в ускорениях [Ulomov et al., 1998].

Использование инструментальной шкалы MSK-64 не позволило стыковать карты в баллах с картами в ускорениях для соседних стран. Поэтому оценки сейсмической интенсивности в баллах пересчитывались в ускорения по данным работы [Аптикаев, Шебалин, 1988], которые вошли и в ныне действующий ГОСТ Р 575462017. Чтобы учесть тот факт, что инструментальная шкала MSK-64 относится к нижним границам интенсивностей, в [СП 14.13330.2014] было записано, что значения ускорений для расчетов должны быть «не ниже 100, 200, 400 см/ $\mathrm{c}^{2}$ для интенсивностей 7, 8, 9 баллов соответственно». Но ничего при этом не изменилось. Проектировщики по-прежнему использовали заниженные в полтора раза значения ускорений. 


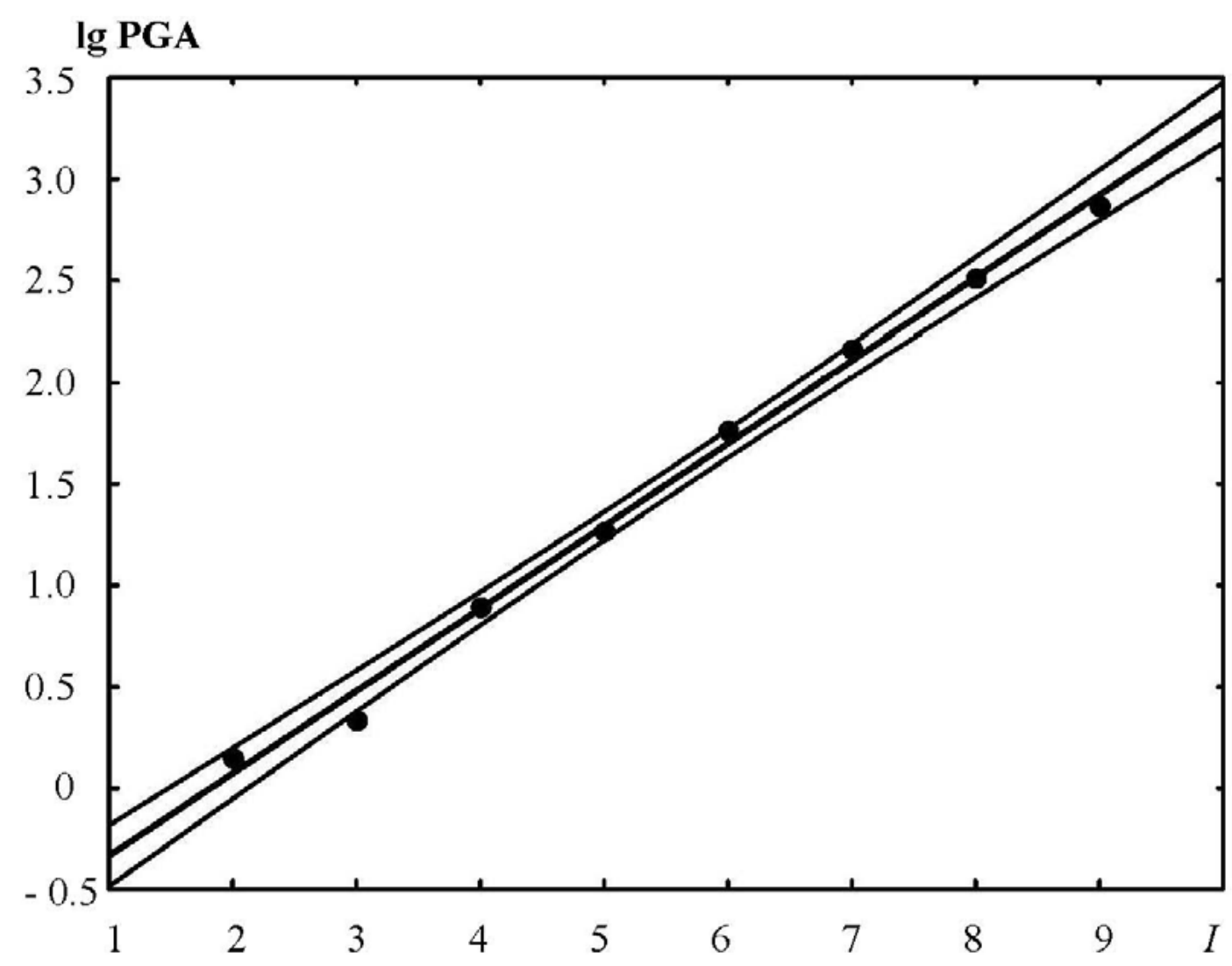

Рис. 1. Соотношение между центрами распределений логарифма пикового ускорения и сейсмической интенсивностью I. Тонкими линиями показан 95\% уровень доверия. По работе [Аптикаев и др., 2008]. Использовано 1554 акселерограммы. /

Fig. 1. Relationship between the centers of distribution of the peak acceleration logarithm and seismic intensity I. Thin lines show a 95\% confidence level. According to [Aptikaev et al., 2008]. Used 1554 accelerograms.

Имелись в версии 2018 года и противоречия. При заниженных уровнях ускорений указывалось, что уровни спектров не должны быть ниже реальных значений. В проекте [СП 14.13330.2019] это противоречие устранено и предлагается пользоваться только заниженными значениями. Ранее для наиболее ответственных объектов коэффициенты ответственности $\kappa_{\text {о }}$ (которые все равно не достигали правильного уровня), в новых нормах уменьшены в полтора раза.

Но и этого мало. Составители норм не считаются с условиями построения карт ОСР. Например, для карты ОСР-С вероятность превышения уровня воздействия в баллах составляет 1\%. Это значит, что, например, для интенсивности 8 баллов значения воздействий выше 8,5 баллов составляют $1 \%$. Этому $1 \%$ будут соответствовать воздействия уже в 9 баллов. Проект новых норм требует вести расчеты не на верхнюю границу диапазона ускорения, и даже не на среднее значение, а на нижнюю границу диапазона, то есть для нашего примера, на 7,5 баллов. Итак карты ОСР оперируют превышением расчетной интенсивности для карты С 1\%, а нормам соответствует превышение уровня ускорений 99\%! Аналогичная ситуация наблюдается и для других интенсивностей и для карт В и А.

Имеются неточности и в оценках уровня превышения сейсмических воздействий в картах ОСР. Составители оперируют П-образной формой распределения 
интенсивности. И оценки превышения $10 \%, 5 \%, 1 \%$ на картах А, В, С соответствуют этой дискретной модели. На самом деле оценки степени повреждений зданий распределены по нормальному закону. Заметим, что и распределение степеней повреждения при конкретном землетрясении также подчиняется нормальному закону [Шебалин, 1969; Кулиев, Шебалин, 1970; Поляков и др., 1982; Ципенюк, 1988; Schenkova, Prochazkova, 1981; Bottari et al., 1986; Velkov, 1989]. А в инженерном диапазоне сейсмическая интенсивность определяется в основном по степени повреждения зданий. Значит, аппроксимация распределения интенсивности П-образным законом также вносит определенную погрешность в оценку вероятности превышения некоторого заданного уровня воздействий. Стандартное отклонение оценки воздействий в баллах при обследовании многих зданий составляет 0,4 балла. Значит, в реальности, например, для карты ОСР-С вероятность превышения уровня воздействий составляет не $1 \%$, а 20\%. Более того, в области заданного балла присутствует $29 \%$ вероятность более высокой интенсивности.

Составители строительных норм совершенно не интересуются достижениями инженерной сейсмологии, учитываемыми в нормах других стран. Расчетные ускорения по нашим нормам существенно ниже имеющихся в зарубежных документах (таблица 2). В таблице приведены инструментальные оценки ускорений шкалы MSK-64 и шкал семейства Меркалли, созданных после 1964 г. ММ-99 - инструментальная часть шкалы Меркалли, модернизированной в 1993 г. [Wald et al., 1999; Shake..., 2006], шкала MCS, до сих пор используемая в Италии [Faenzan, Michelini, 2010], китайская шкала CSIS-99 [Chen et al., 1999], SHA-97 - инструментальная часть шкалы ОСР-97 [Уломов, 1994]. В шкале Республики Казахстан [Методический.., 2015] в среднем придерживались принятому за рубежом шагу шкалы в 2,15 раза на балл, но допускалось различие в шагах для различных интенсивностей. Отметим, что корреляция ускорений и интенсивности практически во всех странах производится с нарушением правил обработки экспериментальных данных: считается, что ошибкам подвержена только одна из сравниваемых величин. Это приводит к заметному занижению наклона аппроксимирующей прямой. Особенно велики ошибки в инженерной сейсмологии, где разброс данных велик, а используемый диапазон интенсивностей всего три балла [Аптикаев, 1961]. У кого ось абсцисс в ускорениях, у того прирост амплитуды на балл завышен (шкала MCS), у кого - в баллах - занижен (шкала МM). Большие ошибки связаны с неверным предположением об удвоении амплитуды на балл (шкалы MSK-64 и CSIS-99). В США для расчетов динамическим методом используются медианные значения ускорений по записям сильных движений, умноженные в полтора раза. У нас - противоположная тенденция. В Eurocode 8 спектры задаются два спектра в зависимости от магнитуды, а у нас магнитуда вообще не принимается во внимание. Во многих публикациях отмечается, что при высоких интенсивностях ускорения на рыхлых грунтах даже меньше, чем на грунтах скальных, хотя интенсивность все-таки выше на рыхлых грунтах [Neumann, 1954; Duke et al., 1972; Trifunac, 1976; Chiaruttini et al., 1979; McGuire, Barnhard, 1979; Campbell, 1981; Joyner, Boore, 1981]. По эмпирическим данным рост интенсивности на рыхлых грунтах объясняется резким увеличением продолжительности колебаний [Arias, 1970; Аптикаев, 2012], фактором, у нас не учитываемым. В нормах США, Ирана и других стран значения коэффициента динамического усиления принимаются на основании обработки эмпирических данных, при определенных условиях достигающие значения $\beta=4$ [Iranian..., 2005; Yu, Chock, 2010]. Среднее 
Таблица 2. / Table 2.

Ускорения для различных интенсивностей в нормах различных стран. / Accelerations for various intensities in the building codes of different countries.

\begin{tabular}{|l|c|c|c|}
\hline \multirow{2}{*}{ Шкала / Scale } & \multicolumn{3}{|c|}{ Интенсивность в баллах / Intensity in } \\
& \multicolumn{3}{|c|}{ роints } \\
\cline { 2 - 4 } & $\mathbf{7}$ & $\mathbf{8}$ & $\mathbf{9}$ \\
\hline MSK-64 & 100 & 200 & 400 \\
\hline ММ-99 & 247 & 470 & 898 \\
\hline SНА-97 & 130 & 280 & 600 \\
\hline Шкала PК / Scale RK & 120 & 270 & 600 \\
\hline ГОСТ P 57546-2017 / GOST R 57546-2017 & 110 & 280 & 700 \\
\hline
\end{tabular}

значение этого коэффициента по результатам обработки тысяч акселерограмм составляет $\beta=3,6$ [Mikhailova, Aptikaev, 1996].

Составители новых норм не учитывают и оценок сейсмических воздействий, полученных в других организациях нашей страны. Значение коэффициента динамического усиления $\beta=3,2$ получено специалистами в области атомной энергетики [РБ-006-98]. В новых СП по-прежнему используется величина $\beta=2,5$, полученная не путем осреднения реальных значений, а путем осреднения значений на фиксированных частотах. Такой прием противоречит правилам обработки эмпирических данных. В НИУ МГСУ [СТО..., 2015] отмечается зависимость сейсмического воздействия от длительности и указывается, что для заданной интенсивности при малой длительности амплитуда ускорения может быть весьма высокой. Влияние продолжительности колебаний на повреждаемость сооружений учитывается в нормах атомной энергетики [РБ 006-98]. А в строительных нормах длительность колебаний полностью игнорируется и даже записано, что для составления синтетической акселерограммы достаточно знать только спектр.

Наиболее перспективный путь повышения надежности расчетов сейсмостойкости - переход на энергетические характеристики сейсмических воздействий. Об этом говорил еще в 1956 году американский ученый C.W. Housner. Этой проблемой занимались J.A. Blume, N.M. Newmark, V.V. Bertero и многие другие ученые США, Италии, Японии. В нашей стране также энергетические характеристики воздействий развиваются известными учеными Ю.Л. Рутманом, А.А. Петровым, А.М. Уздиным, М.А. Клячко, А.А. Долгой и другими. Согласно ГОСТ Р 57546-2017 переход задания воздействий от ускорений к энергетическим характеристикам снизит стандартное отклонение для корреляции воздействий со степенью повреждений в 2,5 раза.

\section{ВывОАЫ}

Оценки уровня сейсмических воздействий в отечественных нормах ниже реальных значений. В проекте последних строительных норм и правил этот уровень оказался еще более заниженным. Проект совершенно не учитывает достижений инженерной сейсмологии за рубежом и в нашей стране. Даже не учитываются нормативные документы других российских организаций и ведомств. Занижение расчетных уровней сейсмических воздействий приводило и будет приводить к неоправданным 
жертвам и материальному ущербу при будущих землетрясениях. Необходимо разработать методы расчета сейсмостойкости на основании ожидаемых энергетических характеристик сейсмического движения грунта.

\section{^итература}

1.Аптикаев Ф.Ф. Инструментальная шкала сейсмической интенсивности [Текст]. / Ф. Ф. Аптикаев; Российская акад. наук, Ин-т физики Земли им. О.Ю. Шмидта. - М.: Наука и образование, 2012. - 175 с.

2.Аптикаев Ф.Ф., Шебалин Н.В. Уточнение корреляций между уровнем макросейсмического эффекта и динамическими параметрами движения грунта. // Исследования по сейсмической опасности (Вопросы инженерной сейсмологии). - 1988. - Вып. 29. - С. 98 107.

3.Аптикаев Ф.Ф., Эртелева О.О., Мокрушина Н.Г. Категория сейсмических шкал семейства Меркалли. // Вулканология и сейсмология. - 2008. - №3. - С. 74-78.

4.ГОСТ Р 57546-2017. Национальный стандарт Российской Федерации Землетрясения. Шкала сейсмической интенсивности. - М.: Стандартинформ, 2017. - 28 с.

5.Кулиев Ф.Т., Шебалин Н.В. О возможности статистической интерпретации балльности. // Сильные землетрясения Средней Азии и Казахстана. - Душанбе: Дониш, 1970. - Вып. 1. - С. 94-118.

6.Медведев С.В. Международная шкала сейсмической интенсивности // Сейсмическое районирование СССР. - М.: Наука, 1968. - С. 151 - 162.

7.Медведев С.В. Определение интенсивности колебаний. // Вопросы инженерной сейсмологии. - 1978. - Вып. 19. - С. 108-116.

8.Методический документ архитектуры, градостроительства и строительства. Рекомендации по определению сейсмических нагрузок, соответствующих инженерно-геологическим и сейсмологическим условиям Республики Казахстан. - Астана. $-2015 .-113$ с.

9.Поляков С.В., Жаров А.М., Мартемьянов А.И., Ширин В.В. Газлийское землетрясение 1976 года. Инженерный анализ последствий. - 1982. - С. 104-112.

10. РБ-006-98. Определение исходных сейсмических колебаний грунта для проектных основ. - М.: Госатомнадзор России, 2000. - 76 с.

11. Седов Л.И. Методы подобия и размерности в механике. / 10-е изд., доп. - М.: Наука, 1987. $-430 \mathrm{c}$.

12. СНИП-ІІ-7-81*. 2013.

13. СП 14.13330.2014. Строительство в сейсмических районах.

14. СП 14.13330.2019. Строительство в сейсмических районах (проект).

15. СТО НИУ МГСУ 2015. Сейсмостойкость зданий и сооружений. Расчетные положения. - Москва. $-2015 .-20$ с.

16. Уломов В.И. Районирование сейсмической опасности на территории России. // Федеральная система сейсмологических наблюдений и прогноза землетрясений. Информационно-аналитический бюллетень Министерства Российской Федерации по чрезвычайным ситуациям. - 1994. - Т. 1. №1, январь - март 1994 г. - С. 41-52.

17. Ципенюк И.Ф. Повреждаемость и надежность крупнопанельных зданий при сейсмических воздействиях. // Вопросы инженерной сейсмологии. - 1988. - Вып. 29. - С. 141153.

18. Шебалин Н.В. Распределение степеней повреждения зданий и использование его для оценки балльности. // Сейсмическая шкала и методы измерения сейсмической интенсивности. - 1975. - С. 87-109.

19. Arias A. A Measure of Earthquake Intensity // Seismic Design for Nuclear Power Plants. / R.J. Hansen, ed. - MIT Press, Cambridge, Massachusetts. - 1970. - pp. 438-483.

20. Bommer J.J., Martinez-Pereira A. Strong-motion parameters: definition, usefulness and predictability. // Proceedings of the XII World Conference on Earthquake Engineering (Auckland, January 1 - February 4, 2000). - Auckland, New Zealand, 2000. - Paper No. 0206. 
21. Bottari A., Corsanego A., Guedice E., Mangeri M. Some problems in the MSK-scale applications: Use of the quantitative definitives in detailed damage assessment. // Ann. Geophys. Bo. - 1986. - Vol. 4. No.2. - pp. $191-200$.

22. Bureau G.J. Near-source peak ground acceleration. // Earthquake Notes. - 1981. - Vol. 52. No.1. - p. 81.

23. Campbell K.W. Near-source attenuation of peak horizontal acceleration. // Bulletin of the Seismological Society of America. - 1981. - Vol. 71. No.6. - pp. 2039-2070.

24. Chen D., Shi Z., Xu Z., Gao G., Nian J., Xiao c., Feng Y. China Seismic Intensity Scale. // General Administration of Quality Supervision, Inspection and Quarantine of R.P.C. - 1999.

25. Chiaruttini C., Crosila F., Siro L. Some maximized acceleration analysis of the 1976 Friuli earthquakes. // Boll. Geof. Teor. Appl. - 1979. - Vol. XXI. - pp. 38-52.

26. Duke C.M. et al. Effects of site classification and distance on instrumental indices in the San Fernando earthquake. Rpt. UCLA-ENG-7247. - Los Angeles. - 1972. - 50 p.

27. Faenzan L., Michelini A. Regression analysis of MCS intensity and ground motion parameters in Italy and its application in ShakeMap. // Geophysical Journal International. - Vol. 180. Issue 3. - 2010. - pp. 1138-1152. https://doi.org/10.1111/j.1365-246X.2009.04467.x

28. Hanks T.S., Johnson D.A. Geophysical assessment of peak accelerations. // Bulletin of the Seismological Society of America. - 1976. - Vol. 66. No.3. - pp. 959-968.

29. Iranian Code of Practice for Seismic Resistant Design of Buildings. Standard № 2800. // Building and Housing Research Center (BHRC). - 2005.

30. Jaiswal K., Wald D.J., Bausch D., Marano K.D. Earthquake Impact Scale. // Natural Hazard Review, ASCF, August 2011.

31. Joyner W.B., Boore D.M. Peak horizontal acceleration and velocity from strong-motion records including records from the 1979 Imperial Valley, California, earthquake. // Bulletin of the Seismological Society of America. - 1981. - Vol. 71. No.6. - pp. 2011-2058.

32. McGuire R.K. and Barnhard T.P. The usefulness of ground motion duration in predicting the severity of seismic shaking. Preprint. $-1979 .-17 \mathrm{p}$.

33. Mikhailova N.N., Aptikaev F.F. Some Correlation Relations between Parameters of Seismic Motions. // Journal of Earthquake Prediction Research. - Moscow-Beijing. - 1996. V. 5. No.2. - pp. 257-267.

34. Neumann R. Earthquake intensity and related ground motion. - Seattle, Univ. Wash. Press, 1954. $-77 \mathrm{p}$.

35. Trifunac M.D. Preliminary analysis of the peaks strong earthquake ground motiondependece of peaks on earthquake magnitude, epicentral distance, and recording site conditions. // Bulletin of the Seismological Society of America. - 1976. - V. 66. No.1. - pp. 189-219.

36. Schenkova Z., Prochazkova D. Determination of representative intensity values of individual localities. // Gerl. Beitr.Geofys. - Leipzig. - Vol. 90. - 1981.

37. Shake Map Manual, Version 1.0 6/19/06. // ADVANCED NATIONAL SEISMIC SYSTEM / David J. Wald, Bruce C. Worden, Vincent Quitoriano, and Kris L. Pankow. - USGS. - 2006. - 156 p. - https://pubs.usgs.gov/tm/2005/12A01/

38. Ulomov V. et al. Working Group of GSHAP, Region 7, 1988, "Seismic Hazard of Northern Eurasia". - http:/www.seismo2009.ethz.ch/gshap/neuurasia/report.html

39. Velkov M. The Armenia-USSR earthquake December 7, 1988. - Inst. Earthq. Eng. and Eng. Seism: Skopje. $-1989 .-70$ p.

40. Wald D.J., Quitoriano V., Heaton T.H., Kanamori H. Relationship between Peak Ground Acceleration, Peak Ground Velocity and Modified Mercalli Intensity in California. // Earthquake Spectra. - Vol. 15. No. 3. - 1999. - pp. 557-564.

41. Yamada M., Olsen A., Heaton T. Statistical features of short- and long-period near-source ground motion. // Bulletin of the Seismological Society of America. - 2009. - V. 99. No.6. - pp. 3264-3274. DOI: 10.1785/0120090067

42. Yu G., Chock G.K. Comparison of the USA, China and Japan Seismic Design Procedures/ China-USA Symposium for the Advancement of Earthquake Sciences and Hazard Mitigation Practices/Beijing, China. - 2010. 


\section{References}

1. Aptikaev F.F. Instrumental scale of seismic intensity. Science and education publishing. 2012. 176 p. (In Russ.)

2. Aptikaev F.F., Shebalin N.V. Clarification of the correlation between level of macroseismic effect and dynamic parameters of ground motion. Problems of engineering seismology. 1988. Vol. 29. pp. 98-107. (In Russ.)

3. Aptikaev F.F., Erteleva O.O., Mokrushina N.G. Category seismic scales family of Mercalli. Volcanology and seismology. 2008. No. 3. pp. 74-78. (In Russ.)

4. GOST R 57546-2017. National standard of the Russian Federation of the Earthquake. Scale of seismic intensity. Moscow. Standartinform. 2017. 28 p. (In Russ.)

5. Kuliev, F.T., Shebalin N.V. About the possibility of a statistical interpretation of intensity. Strong earthquake in Central Asia and Kazakhstan. Dushanbe. Donish. 1970. Vol. 1. pp. 94-118. (In Russ.)

6. Medvedev S.V. International scale of seismic intensity. Seismic zoning of the USSR. Moscow. Nauka. 1968. pp. 151-162. (In Russ.)

7. Medvedev S.V. Determination of intensity oscillations. Questions of engineering seismology. 1978. Vol. 19. pp. 108-116. (In Russ.)

8. Methodological document of architecture, urban planning and construction. Recommendations for determining seismic treatment, relevant engineering-geological and seismological conditions of the Republic of Kazakhstan. Astana. 2015. 113 p. (In Russ.)

9. Polyakov S.V., Zharov, A.M., Martem'yanov, A.I., Shirin V.V. Gasli earthquake of 1976. Engineering analysis of the consequences. 1982. pp. 104-112.

10. RB-006-98. Determination of initial seismic vibrations of soil for design of foundations. Moscow. Gosatomnadzor of Russia. 2000. 76 p. (In Russ.)

11. Sedov L.I. Methods of similarity and dimension in mechanics. Moscow. Nauka. 1987. 430 p. (In Russ.)

12. SNIP-II-7-81*. 2013. (In Russ.)

13. SP 14.13330.2014. Construction in seismic areas. (In Russ.)

14. SP 14.13330.2019. Construction in seismic areas (draft). (In Russ.)

15. STO NIU MGSU 2015. The seismic stability of buildings and structures. The design position. Moscow. 2015. 20 p. (In Russ.)

16. Ulomov V.I. Seismic hazard zoning in Russia/Federal system of seismological observations and earthquake prediction. Information-analytical Bulletin of the Ministry of the Russian Federation for emergency situations. 1994. Vol. 1. No. 1, January-March. 1994. pp. 4152. (In Russ.)

17. Tsipenyuk I.F. Damageability and reliability of large-panel buildings under seismic forces. Questions of engineering seismology. 1988. Vol. 29. pp. 141-153. (In Russ.)

18. Shebalin N.V. The distribution of the degrees of damage to buildings and use it to evaluate the intensity. Seismic scale and methods of measuring seismic intensity. 1975. pp. 87-109. (In Russ.)

19. Arias A. A Measure of Earthquake Intensity. Seismic Design for Nuclear Power Plants. R.J. Hansen, ed. MIT Press, Cambridge, Massachusetts. 1970. pp. 438-483.

20. Bommer J.J., Martinez-Pereira A. Strong-motion parameters: definition, usefulness and predictability. Proceedings of the XII World Conference on Earthquake Engineering (Auckland, January 1 - February 4, 2000). Auckland, New Zealand. 2000. Paper No. 0206.

21. Bottari A., Corsanego A., Guedice E., Mangeri M. Some problems in the MSK-scale applications: Use of the quantitative definitives in detailed damage assessment. Ann. Geophys. Bo. 1986. Vol. 4. No.2. pp. 191-200.

22. Bureau G.J. Near-source peak ground acceleration. Earthquake Notes. 1981. Vol. 52. No.1. $81 \mathrm{p}$. 
23. Campbell K.W. Near-source attenuation of peak horizontal acceleration. Bulletin of the Seismological Society of America. 1981. Vol. 71. No.6. pp. 2039-2070.

24. Chen D., Shi Z., Xu Z., Gao G., Nian J., Xiao c., Feng Y. China Seismic Intensity Scale. General Administration of Quality Supervision, Inspection and Quarantine of R.P.C. 1999.

25. Chiaruttini C., Crosila F., Siro L. Some maximized acceleration analysis of the 1976 Friuli earthquakes. Boll. Geof. Teor. Appl. 1979. Vol. XXI. pp. 38-52.

26. Duke C.M. et al. Effects of site classification and distance on instrumental indices in the San Fernando earthquake. Rpt. UCLA-ENG-7247. Los Angeles. 1972. 50 p.

27. Faenzan L., Michelini A. Regression analysis of MCS intensity and ground motion parameters in Italy and its application in ShakeMap//Geophysical Journal International, Volume 180, Issue 3, March 2010. pp. 1138-1152. https://doi.org/10.1111/j.1365-246X.2009.04467.x

28. Hanks T.S., Johnson D.A. Geophysical assessment of peak accelerations. Bulletin of the Seismological Society of America. 1976. Vol. 66. No.3. pp. 959-968.

29. Iranian Code of Practice for Seismic Resistant Design of Buildings. Standard № 2800. Building and Housing Research Center (BHRC). 2005.

30. Jaiswal K., Wald D.J., Bausch D., Marano K.D. Earthquake Impact Scale. Natural Hazard Review, ASCF, August. 2011.

31. Joyner W.B., Boore D.M. Peak horizontal acceleration and velocity from strong-motion records including records from the 1979 Imperial Valley, California, earthquake. Bulletin of the Seismological Society of America. 1981. Vol. 71. No.6. pp. 2011-2058.

32. McGuire R.K. and Barnhard T.P. The usefulness of ground motion duration in predicting the severity of seismic shaking. Preprint. 1979. $17 \mathrm{p}$.

33. Mikhailova N.N., Aptikaev F.F. Some Correlation Relations between Parameters of Seismic Motions. Journal of Earthquake Prediction Research. Moscow-Beijing. 1996. V. 5. No.2. pp. 257-267.

34. Neumann R. Earthquake intensity and related ground motion. Seattle, Univ. Wash. Press. 1954. $77 \mathrm{p}$.

35. Trifunac M.D. Preliminary analysis of the peaks strong earthquake ground motiondependece of peaks on earthquake magnitude, epicentral distance, and recording site conditions. Bulletin of the Seismological Society of America. 1976. V. 66. No.1. pp. 189-219.

36. Schenkova Z., Prochazkova D. Determination of representative intensity values of individual localities. Gerl. Beitr.Geofys. Leipzig. Vol. 90. 1981.

37. Shake Map Manual, Version 1.0 6/19/06. ADVANCED NATIONAL SEISMIC SYSTEM. David J. Wald, Bruce C. Worden, Vincent Quitoriano, and Kris L. Pankow. USGS. - 2006. 156 p. https://pubs.usgs.gov/tm/2005/12A01/

38. Ulomov V. et al. Working Group of GSHAP, Region 7, 1988, "Seismic Hazard of Northern Eurasia". http:/www.seismo2009.ethz.ch/gshap/neuurasia/report.html

39. Velkov M. The Armenia-USSR earthquake December 7, 1988. Inst. Earthq. Eng. and Eng. Seism: Skopje. 1989. 70 p.

40. Wald D.J., Quitoriano V., Heaton T.H., Kanamori H. Relationship between Peak Ground Acceleration, Peak Ground Velocity and Modified Mercalli Intensity in California//Earthquake Spectra. 1999. Vol. 15. No. 3. pp. 557-564.

41. Yamada M., Olsen A., Heaton T. Statistical features of short- and long-period nearsource ground motion. Bulletin of the Seismological Society of America. 2009. V. 99. No.6. pp. 3264-3274. DOI: $10.1785 / 0120090067$

42. Yu G., Chock G.K. Comparison of the USA, China and Japan Seismic Design Procedures/ China-USA Symposium for the Advancement of Earthquake Sciences and Hazard Mitigation Practices. Beijing, China. 2010. 